\title{
Extremal discs in almost complex spaces
}

\author{
Hervé GAUSSIER AND JAE-CHEON JoO
}

\begin{abstract}
We give a necessary condition for the existence of extremal pseudoholomorphic discs on some domains in $\left(\mathbb{R}^{2 n}, J\right)$ where $J$ is a small almost complex deformation of the standard complex structure.
\end{abstract}

Mathematics Subject Classification (2010): 32F45 (primary); 32G05, 32H99, $53 \mathrm{C} 15$ (secondary).

\section{Introduction}

This paper deals with the study of extremal discs in almost complex manifolds. In the seminal paper [14] L. Lempert proved that every convex domain $\Omega$ admits a singular foliation by such discs which are proper and smooth up to the boundary in case the domain $\Omega=\{\rho<0\}$ is smooth. The proof of existence of the foliation relies on an analytic characterization of extremal discs: if $f$ is an extremal disc in $\Omega$ then $f$ is stationary, meaning that there exists a positive function $p$ defined on the unit circle $\partial \Delta$ such that the function $\zeta \mapsto \zeta p(\zeta) d \rho(f)$ admits a holomorphic extension to the unit disc $\Delta$. Important applications of extremal discs concern the study of moduli spaces (see for instance [16] and [3]) or the existence of a Green function for the homogeneous Monge-Ampère equation (see for instance $[14,20]$ ). Stationary discs are natural global biholomorphic invariants of complex manifolds with boundary. The smooth extension of stationary discs is an essential ingredient in boundary values problems such as the Fefferman extension theorem, as proved by L. Lempert [15] in the complex setting (see [6,8] for almost complex manifolds).

Lempert's results were generalized to strongly pseudoconvex domains in the complex Euclidean space by E. Poletsky [21] and M. Y. Pang [19] using variational principles. Finally A. Tumanov [24] gave a geometric characterization of the stationarity condition: a holomorphic disc $f$ attached to the boundary of a domain $\Omega$ is stationary if and only if $f$ admits a meromorphic lift to the cotangent bundle with boundary attached to the conormal bundle over $\Omega$.

The second author's research is supported by the Grant KRF-2008-357-C00006 of the Korea Research Foundation.

Received July 20, 2009; accepted in revised form November 13, 2009. 
Although there exist foliations by stationary discs in the almost complex setting $[7,23]$, we first prove in this paper that in contrast with the complex setting governed by Lempert's theory there exist extremal discs which are not stationary discs. Our main goal is to provide a new analytic and geometric characterization of extremal discs for smooth almost complex structures which are close to the standard complex structure on the closure of a given convex domain in the Euclidean space $\mathbb{R}^{2 n}$. We deduce from this characterization the existence of an extremal disc through each point in any direction.

\subsection{Organization of the paper}

Our approach is based on a variational principle in the spirit of [19,21]. Throughout the paper we consider a bounded domain $\Omega=\{\rho<0\}$ contained in the almost complex manifold $\left(\mathbb{R}^{2 n}, J\right)$, where $J$ is a smooth almost complex structure on $\mathbb{R}^{2 n}$. We assume that $J$ is sufficiently close to the standard structure $J_{\text {st }}$ on some fixed neighbourhood of the closure of $\Omega$ for an appropriate norm to be specified in the different sections.

In Section 2 we give an example of a proper extremal disc in the unbounded representation of the ball, endowed with a non-integrable almost complex structure, which is not stationary. Although the domain is not relatively compact the closed extremal disc is bounded and the domain is strongly pseudoconvex in a neighborhood of the closure of the disc.

The remaining part of the paper consists in finding a characterization of closed extremal discs, giving a substitute to the stationarity condition introduced by L. Lempert.

In Section 3 we study the properties of $J$-holomorphic variational vector fields. These will be essential to characterize extremal discs.

In Section 4 we focus on extremal discs. We prove in Proposition 4.1 that every closed extremal disc contained in $\Omega$ is proper. In Theorem 4.3 we prove that every closed extremal disc contained in $\Omega$ vanishes the first order variations. This technical result will give the necessary condition for a $J$-holomorphic disc to be extremal.

In Section 5 we find an analogue of the stationarity condition called $J$-stationarity. We prove in Theorem 5.2 that a smooth closed extremal disc embedded into $\Omega$ is $J$-stationary if the structure $J$ is close to the standard structure $J_{\text {st }}$.

Finally in Section 6 we prove that every smooth $J$-stationary disc $f$ is locally extremal if the domain is strongly convex in a neighborhood of $f(\bar{\Delta})$ (see Theorem 6.4).

ACKNOWLEDGEMENTS. We would like to thank Y. J. Choe for communicating his results concerning extremal discs in model almost complex manifolds. 


\section{A non stationary extremal disc}

Throughout this paper, the summation convention will be used.

\subsection{Preliminaries}

We denote by $\mathbb{R}^{2 n}$ the $2 n$-dimensional real Euclidean space with standard coordinate system $\left(x^{1}, \ldots, x^{2 n}\right)$. The complex Euclidean space equipped with complex coordinates $\left(z^{1}, \ldots, z^{n}\right)$ is denoted by $\mathbb{C}^{n}$. The real and complex coordinates satisfy the relation:

$$
z^{j}=x^{2 j-1}+\sqrt{-1} x^{2 j}
$$

for $j=1, \ldots, n$. An almost complex structure on an open set $U$ of $\mathbb{R}^{2 n}$ is a smooth section $J$ of the tangent bundle $T U$ such that

$$
J_{p}^{2}=-\left.I d\right|_{T_{p} U}
$$

for every $p \in U$. We will mention the different precise smoothness assumptions on $J$ in each result of the paper. The standard complex structure will be denoted by $J_{\text {st }}$, that is,

$$
\left(\mathbb{R}^{2 n}, J_{\mathrm{st}}\right)=\mathbb{C}^{n} .
$$

We denote by $\Delta$ the open unit disc in $\mathbb{C}$ with standard complex coordinate $\zeta=$ $x+\sqrt{-1} y$. Let $J$ be an almost complex structure on $\mathbb{R}^{2 n}$. A smooth mapping $f: \Delta \rightarrow \mathbb{R}^{2 n}$ is called a $J$-holomorphic disc if it satisfies the Cauchy-Riemann equation

$$
\frac{\partial f}{\partial x}+J(f) \frac{\partial f}{\partial y}=0
$$

on $\Delta$. Moreover if $f$ is continuous (respectively $C^{\alpha}$-smooth for some real $\alpha>0$ ) up to the boundary, then we call $f$ a closed (respectively $C^{\alpha}$-closed) J-holomorphic disc.

A real-valued smooth function $\rho$ is said to be $J$-plurisubharmonic if

$$
d d_{J}^{c} \rho(X, J X) \geq 0
$$

for every tangent vector $X$, where $d_{J}^{c} \rho(X):=-d \rho(J X)$. In case the inequality is strict whenever $X \neq 0$, we say that $\rho$ is strictly $J$-plurisubharmonic. It is known that $\rho \circ f$ is subharmonic on $\Delta$ if $\rho$ is $J$-plurisubharmonic and $f$ is a $J$-holomorphic disc.

\subsection{Extremal and stationary dises}

Let $\rho$ be a $C^{2}$-smooth function on $\mathbb{R}^{2 n}$, let $\Omega=\{\rho<0\}$ be a bounded domain in $\mathbb{R}^{2 n}$ and let $J$ be a $C^{1}$-smooth almost complex structure on $\mathbb{R}^{2 n}$. 
Definition 2.1. A $J$-holomorphic disc $f: \Delta \rightarrow \Omega$ is extremal if for every $J$ holomorphic disc $g: \Delta \rightarrow \Omega$ such that $g(0)=f(0)$ and $g^{\prime}(0)=\lambda f^{\prime}(0)$ for some $\lambda \geq 0$, it holds that $\lambda \leq 1$.

To describe extremal discs, L. Lempert [14] introduced the notion of stationary discs on strongly convex domain in $\mathbb{C}^{n}$. Let $T^{*} \mathbb{R}^{2 n}$ be the cotangent bundle of $\mathbb{R}^{2 n}$. For a covector $\omega=p_{i} d x^{i}$, we consider the coordinates $\omega:\left(x^{1}, \ldots, x^{2 n}, p_{1}, \ldots, p_{2 n}\right)$ in $T^{*} \mathbb{R}^{2 n}$. The canonical lift of $J$ (in the terminology of [23], see [11] for a precise definition and properties of this lifted structure) is the almost complex structure on $T^{*} \mathbb{R}^{2 n}$ defined by:

$$
\widetilde{J}:=\left(\begin{array}{cc}
J & 0 \\
L & J^{t}
\end{array}\right)
$$

where for $i, j=1, \ldots, 2 n$,

$$
L_{i j}=-\frac{1}{2} p_{a}\left\{-\frac{\partial J_{i}^{a}}{\partial x^{j}}+\frac{\partial J_{j}^{a}}{\partial x^{i}}+J_{l}^{a}\left(\frac{\partial J_{i}^{l}}{\partial x^{m}} J_{j}^{m}-\frac{\partial J_{j}^{l}}{\partial x^{m}} J_{i}^{m}\right)\right\} .
$$

Definition 2.2. A properly embedded closed $J$-holomorphic disc $f: \Delta \rightarrow \Omega$ is a stationary disc if there exists a continuous map $u: \bar{\Delta} \rightarrow \mathbb{R}^{2 n}$, of class $C^{1}$ on $\Delta$, and a continuous positive function $p$ on $\partial \Delta$ such that

$$
\tilde{f}:=(f, u)
$$

is $\widetilde{J}$-holomorphic on $\Delta$ and

$$
u(\zeta)=\zeta p(\zeta) \mathrm{d} \rho(f(\zeta))
$$

for every $\zeta \in \partial \Delta$. The map $\tilde{f}$ is called the holomorphic lift of $f$.

Here the multiplication of a covector $\omega=p_{i} d x^{i}$ by $\zeta=x+\sqrt{-1} y$ is defined by $\zeta \omega=\left(x p_{j}+y J_{j}^{i} p_{i}\right) d x^{j}$.

\section{Remark 2.3.}

1. A.Tumanov [24] generalized L. Lempert's idea, considering meromorphic lifts of discs to the cotangent bundle, which are attached to the conormal bundle. In definition 2.2 we define stationary discs in almost complex spaces in terms of holomorphic lifting. In [6] and [23], following [24], the authors used meromorphic lifts instead of holomorphic lifts to define stationary discs. They call a closed $J$-holomorphic disc $f$ which is attached to the boundary stationary if there exists a continuous lifted map $(f, \tilde{u}): \bar{\Delta} \backslash\{0\} \rightarrow T^{*} \mathbb{R}^{2 n}$ which is $\widetilde{J}$-holomorphic on $\Delta \backslash\{0\}$, such that $0 \neq \tilde{u}(\zeta)$ is parallel with $d \rho(f(\zeta))$ for $\zeta \in \partial \Delta$ and has singularity at the origin of order at most 1 . This definition coincides with Definition 2.2. Indeed it follows from the definition of $\tilde{J}$ that if $u:=\zeta \tilde{u}$, then $\tilde{f}=(f, u)$ is still $\widetilde{J}$-holomorphic. Applying the removable singularity theorem, $\tilde{f}$ becomes a holomorphic lift of $f$. 
2. Spiro [22] proved that the conormal bundle $\mathcal{N}:=\{t d \rho(z): t \in \mathbb{R} \backslash\{0\}, z \in$ $\partial \Omega$ \} is a totally real submanifold in $T^{*} \mathbb{R}^{2 n}$ with respect to $\widetilde{J}$, if $\Omega$ is strongly pseudoconvex with respect to $J$. Since $(f, \tilde{u})$ is $\tilde{J}$-holomorphic on $\Delta \backslash\{0\}$ and is attached to the conormal bundle, one can see that $(f, \tilde{u})$ and hence $\tilde{f}$ are smooth up to $\partial \Delta$ by a standard elliptic bootstrapping method. For more details on this subject, see $[6,23]$.

If $J=J_{\mathrm{st}}$, then $(f, u)$ is $\widetilde{J}$-holomorphic if and only if both $f$ and $u$ are holomorphic in the standard sense. If $J$ is a general almost complex structure this is no more true. However we have:

Proposition 2.4. Let $f: \Delta \rightarrow \mathbb{R}^{2 n}$ be a J-holomorphic disc. Suppose $(f, u)$ : $\Delta \rightarrow T^{*} \mathbb{R}^{2 n}$ is $\widetilde{J}$-holomorphic. Then the complex pairing $\left(u \mid f^{\prime}\right)$ defined by

$$
\left(u \mid f^{\prime}\right):=u_{i} \frac{\partial f^{i}}{\partial x}-\sqrt{-1} J_{j}^{i}(f) u_{i} \frac{\partial f^{j}}{\partial x}
$$

is a holomorphic function on $\Delta$.

Proof. For the sake of simplicity, we will use the notation $h_{x}$ and $h_{y}$ instead of $\partial h / \partial x$ and $\partial h / \partial y$ for a function $h$ on $\Delta$. Let

$$
U:=\operatorname{Re}\left(u \mid f^{\prime}\right)=u_{i} f_{x}^{i}
$$

and

$$
V:=\operatorname{Im}\left(u \mid f^{\prime}\right)=-J_{j}^{i}(f) u_{i} f_{x}^{j}=-u_{i} f_{y}^{i} .
$$

Since $(f, u)$ is $\widetilde{J}$-holomorphic,

$$
u_{i x}+J_{i}^{j} u_{j y}-u_{k} L_{i j}^{k} f_{y}^{j}=0
$$

and

$$
u_{i y}-J_{i}^{j} u_{j x}+u_{k} L_{i j}^{k} f_{x}^{j}=0
$$

for every $i=1, \ldots, 2 n$, where

$$
L_{i j}^{k}=\frac{1}{2}\left\{-\frac{\partial J_{i}^{k}}{\partial x^{j}}+\frac{\partial J_{j}^{k}}{\partial x^{i}}+J_{l}^{k}\left(\frac{\partial J_{i}^{l}}{\partial x^{m}} J_{j}^{m}-\frac{\partial J_{j}^{l}}{\partial x^{m}} J_{i}^{m}\right)\right\} .
$$

Differentiating the Cauchy-Riemann equation for $f$, we get

$$
f_{x x}^{i}+J_{j}^{i} f_{y x}^{j}+\frac{\partial J_{j}^{i}}{\partial x^{k}} f_{y}^{j} f_{x}^{k}=0
$$

and

$$
f_{y y}^{i}-J_{j}^{i} f_{x y}^{j}-\frac{\partial J_{j}^{i}}{\partial x^{k}} f_{x}^{j} f_{y}^{k}=0
$$

for every $i=1, \ldots, 2 n$.

$$
U_{x}-V_{y}=\left(u_{i x} f_{x}^{i}+u_{i y} f_{y}^{i}\right)+u_{i}\left(f_{x x}^{i}+f_{y y}^{i}\right) .
$$


By (2.2) and (2.3),

$$
\begin{aligned}
u_{i x} f_{x}^{i}+u_{i y} f_{y}^{i} & =-J_{i}^{j} u_{j y} f_{x}^{i}+u_{k} L_{i j}^{k} f_{y}^{j} f_{x}^{i}+J_{i}^{j} u_{j x} f_{y}^{i}-u_{k} L_{i j}^{k} f_{x}^{j} f_{y}^{i} \\
& =-\left(u_{i x} f_{x}^{i}+u_{i y} f_{y}^{i}\right)+2 u_{k} f_{x}^{i} f_{y}^{j} L_{i j}^{k}
\end{aligned}
$$

since $L_{i j}^{k}=-L_{j i}^{k}$. Therefore,

$$
u_{i x} f_{x}^{i}+u_{i y} f_{y}^{i}=u_{k} f_{x}^{i} f_{y}^{j} L_{i j}^{k}=u_{k} f_{x}^{i} f_{x}^{l} J_{l}^{j} L_{i j}^{k} .
$$

From (2.4) and (2.5),

$$
u_{i}\left(f_{x x}^{i}+f_{y y}^{i}\right)=\left(\frac{\partial J_{j}^{i}}{\partial x^{k}}-\frac{\partial J_{k}^{i}}{\partial x^{j}}\right) u_{i} f_{x}^{j} f_{y}^{k}=\left(\frac{\partial J_{j}^{i}}{\partial x^{k}}-\frac{\partial J_{k}^{i}}{\partial x^{j}}\right) u_{i} f_{x}^{j} f_{x}^{l} J_{l}^{k} .
$$

Plugging (2.6) with (2.7) and (2.8), we have

$$
U_{x}-V_{y}=u_{k} f_{x}^{i} f_{x}^{l} A_{i l}^{k}
$$

where

$$
A_{i l}^{k}=J_{l}^{j}\left\{L_{i j}^{k}+\left(\frac{\partial J_{i}^{k}}{\partial x^{j}}-\frac{\partial J_{j}^{k}}{\partial x^{i}}\right)\right\} .
$$

Since $A_{i l}^{k}=-A_{l i}^{k}$ from the definition of $L_{i j}^{k}$, it holds that

$$
U_{x}-V_{y}=0 .
$$

Similarly we prove that $U_{y}+V_{x}=0$.

Corollary 2.5. Let $\Omega=\{\rho<0\}$ be a bounded smooth domain in $\mathbb{R}^{2 n}$ defined by a $J$-plurisubharmonic function $\rho$. Suppose $f$ is a stationary disc of $\Omega$ with holomorphic lift $\tilde{f}=(f, u)$. Then the complex pairing $\left(u \mid f^{\prime}\right)$ is a positive constant.

Proof. Let $\zeta=\exp (\sqrt{-1} \theta) \in \partial \Delta$. Since $\rho \circ f$ is subharmonic,

$$
\left.\frac{\mathrm{d}}{\mathrm{d} r} \rho \circ f(r \exp (\sqrt{-1} \theta))\right|_{r=1}=\left\langle\zeta \mathrm{d} \rho(f(\zeta)), f^{\prime}(\zeta)\right\rangle>0
$$

by the Hopf lemma, where $\langle\cdot, \cdot\rangle=\operatorname{Re}(\cdot \cdot \cdot)$ denotes the real pairing between a covector and a vector. Since $\rho \circ f \equiv 0$ on $\partial \Delta$,

$$
\operatorname{Im}\left(\zeta \mathrm{d} \rho(f(\zeta)) \mid f^{\prime}(\zeta)\right)=-\frac{\mathrm{d}}{\mathrm{d} \theta} \rho \circ f(\zeta)=0 .
$$

Therefore, $\left(u \mid f^{\prime}\right)$ is a holomorphic function on $\Delta$ which is positive real-valued on $\partial \Delta$. This yields the conclusion.

Hence multiplying by a suitable positive constant, we may always assume that

$$
\left(u \mid f^{\prime}\right) \equiv 1,
$$

if $f$ is a stationary disc and $\tilde{f}=(f, u)$ is the holomorphic lift of $f$ to the cotangent bundle $T^{*} \mathbb{R}^{2 n}$. 


\subsection{An example of an extremal disc which is not stationary}

When $J=J_{\text {st }}$, it is known that every $C^{1}$-closed embedded extremal disc into a bounded strongly pseudoconvex domain $\Omega$ is proper and stationary by Poletsky [21] and Pang [19]. In the remaining part of this Section we provide a counterexample in the almost complex setting.

For $t>0$, let $J_{t}$ be an almost complex structure on $\mathbb{R}^{6}$ defined by:

$$
J_{t}:=\left(\begin{array}{cccccc}
0 & -1 & 0 & 0 & 0 & t x^{3} \\
1 & 0 & 0 & 0 & t x^{3} & 0 \\
0 & 0 & 0 & -1 & 0 & 0 \\
0 & 0 & 1 & 0 & 0 & 0 \\
0 & 0 & 0 & 0 & 0 & -1 \\
0 & 0 & 0 & 0 & 1 & 0
\end{array}\right)
$$

Let $\Omega=\left\{\rho(z)=\operatorname{Re} z^{1}+\left|z^{2}\right|^{2}+\left|z^{3}\right|^{2}<0\right\} \subset \mathbb{R}^{6}$. Lee [13] showed that $\Omega$ is a homogeneous strongly $J_{t}$-pseudoconvex domain for every $t>0$ and that every strongly pseudoconvex domain with noncompact automorphism group in an almost complex manifold of real dimension 6 is biholomorphic to $\left(\Omega, J_{t}\right)$ for some $t$.

Proposition 2.6. The disc $f(\zeta):=(-1, \zeta, 0)$ is $J_{t}$-holomorphic and extremal in $\Omega$, but not stationary.

Proof. The disc $f$ is obviously smooth up to the boundary. Choe [5] proved that there exists a uniquely determined $J_{t}$-Hermitian invariant metric which coincides with the standard Bergman metric at $(-1,0,0)$ and has a constant negative holomorphic sectional curvature -4 . From the Ahlfors-Kobayashi Schwarz lemma, this implies that $f$ is a $J_{t}$-holomorphic extremal disc in $\Omega$ for every $t>0$.

The canonical lift $\widetilde{J}_{t}$ of $J_{t}$ to $T^{*} \mathbb{R}^{6}$ is given by the $(12 \times 12)$-real matrix:

$$
\widetilde{J}_{t}=\left(\begin{array}{rrrrrrrrrrrr}
0 & -1 & 0 & 0 & 0 & t x_{3} & 0 & 0 & 0 & 0 & 0 & 0 \\
1 & 0 & 0 & 0 & t x_{3} & 0 & 0 & 0 & 0 & 0 & 0 & 0 \\
0 & 0 & 0 & -1 & 0 & 0 & 0 & 0 & 0 & 0 & 0 & 0 \\
0 & 0 & 1 & 0 & 0 & 0 & 0 & 0 & 0 & 0 & 0 & 0 \\
0 & 0 & 0 & 0 & 0 & -1 & 0 & 0 & 0 & 0 & 0 & 0 \\
0 & 0 & 0 & 0 & 1 & 0 & 0 & 0 & 0 & 0 & 0 & 0 \\
0 & 0 & 0 & 0 & 0 & 0 & 0 & 1 & 0 & 0 & 0 & 0 \\
0 & 0 & 0 & 0 & 0 & 0 & -1 & 0 & 0 & 0 & 0 & 0 \\
0 & 0 & 0 & 0 & -\frac{t p_{2}}{2} & -\frac{t p_{1}}{2} & 0 & 0 & 0 & 1 & 0 & 0 \\
0 & 0 & 0 & 0 & \frac{t p_{1}}{2} & -\frac{t p_{2}}{2} & 0 & 0 & -1 & 0 & 0 & 0 \\
0 & 0 & \frac{t p_{2}}{2} & -\frac{t p_{1}}{2} & 0 & 0 & 0 & t x_{3} & 0 & 0 & 0 & 1 \\
0 & 0 & \frac{t p_{1}}{2} & \frac{t p_{2}}{2} & 0 & 0 & t x_{3} & 0 & 0 & 0 & -1 & 0
\end{array}\right) .
$$


Assume by contradiction that $f$ is stationary. Let $\tilde{f}=(f, u)$ be the holomorphic lift of $f$ to $T^{*} \mathbb{R}^{6}$. From the boundary condition (2.1) satisfied by $u$ and from Condition (2.9), we have for $(x, y) \in \partial \Delta$ :

$$
u(x, y)=\left(\frac{x}{2},-\frac{y}{2}, 1,0,0,0\right) .
$$

Let $u=(a, b, 1,0, c, d)$ where $a, b, c$ and $d$ are real functions on $\Delta$ by (2.9). Then

$$
\begin{aligned}
& a_{y}=b_{x}, b_{y}=-a_{x}, \\
& c_{y}=\frac{t b}{2}+t x b_{x}+d_{x}
\end{aligned}
$$

and

$$
d_{y}=\frac{t a}{2}+t x a_{x}-c_{x}
$$

by (2.2) and (2.3). Equalities (2.10) and (2.11) imply that $a(x, y)=x / 2$ and $b(x, y)=-y / 2$ on $\Delta$. Therefore, we have

$$
c_{y}=d_{x}-t y / 4 \text { and } d_{y}=-c_{x}+3 t x / 4
$$

by (2.12) and (2.13). We deduce from (2.14) that $d$ is harmonic and hence $d \equiv 0$ on $\Delta$ by the boundary condition (2.10). Finally, we obtain from (2.14):

$$
c=3 t x^{2} / 8-t y^{2} / 8+C
$$

for some real constant $C$. This contradicts the boundary condition (2.10).

Remark 2.7. The non-stationarity of the $J$-holomorphic extremal disc $f(\zeta)=$ $(-1, \zeta, 0)$ relies on the fact that the space of $J$-holomorphic variational vector fields along $f$ is not stable under multiplication by $J$ (see the next section for a precise definition of holomorphic variational vector fields). One can prove that if this space is stable under multiplication by $J$ then the corresponding extremal disc is stationary.

\section{Holomorphic variational vector fields}

Let $\alpha>2$ be a non-integer real number. Let $J$ be a $C^{\alpha}$-smooth almost complex structure on $\mathbb{R}^{2 n}$. Let $f_{t}$ be a one parameter family of $J$-holomorphic discs into $\left(\mathbb{R}^{2 n}, J\right)$, of class $C^{1}$ in $t \in \mathbb{R}$. Consider for $\zeta \in \Delta$

$$
v(\zeta):=\left.\frac{\mathrm{d}}{\mathrm{d} t} f_{t}(\zeta)\right|_{t=0}
$$

From the $J$-holomorphicity of $f_{t}$, we have

$$
\frac{\partial f_{t}^{i}}{\partial x}+J_{j}^{i}\left(f_{t}\right) \frac{\partial f_{t}^{j}}{\partial y}=0
$$

for every $i=1, \ldots, 2 n$. 
Differentiating (3.1) in $t$ at $t=0$, we get:

$$
\frac{\partial v^{i}}{\partial x}+J_{j}^{i}(f) \frac{\partial v^{j}}{\partial y}+\frac{\partial J_{j}^{i}}{\partial x^{k}}(f) \frac{\partial f^{j}}{\partial y} v^{k}=0
$$

for every $i=1, \ldots, 2 n$, where $f=f_{0}$.

Definition 3.1. Let $f$ be a $J$-holomorphic disc in $\left(\mathbb{R}^{2 n}, J\right)$. A $C^{1}$-smooth mapping $v: \Delta \rightarrow \mathbb{R}^{2 n}$ is called a J-holomorphic variational vector field along $f$ if it satisfies equation (3.2).

We summarize basic properties of $J$-holomorphic variational vector fields.

Proposition 3.2. Let $f: \Delta \rightarrow\left(\mathbb{R}^{2 n}, J\right)$ be a J-holomorphic disc. Then the following holds.

(a) If $u$ and $v$ are J-holomorphic variational vector fields along $f$, then so is $u+v$.

(b) Let $J_{1}$ be an almost complex structure on $\mathbb{R}^{2 m}$ and let $\Phi: U \supset \overline{f(\Delta)} \rightarrow \mathbb{R}^{2 m}$ be a pseudo-holomorphic map defined on an open neighborhood $U$ in $\mathbb{R}^{2 n}$, that is satisfying $d \Phi \circ J=J_{1} \circ d \Phi$. Then $d \Phi(v)$ is a $J_{1}$-holomorphic variational vector field along $\Phi \circ f$ whenever $v$ is a J-holomorphic variational vector field along $f$.

(c) The velocity vector field $f^{\prime}:=\partial f / \partial x$ of $f$ is a J-holomorphic variational vector field along $f$.

(d) Moreover, if $\phi=a+\sqrt{-1} b: \Delta \rightarrow \mathbb{C}$ is a holomorphic function, then so is $\phi f^{\prime}:=a f^{\prime}+b J f^{\prime}$.

A proof of Proposition 3.2 is straightforward from the definition of $J$-holomorphic variational vector fields.

\section{Remark 3.3.}

1. The definition of $J$-holomorphic variational vector fields is related to the complete lift $\widehat{J}$ of $J$ to the tangent bundle $T \mathbb{R}^{2 n}$. Let $\left(x^{i}, q^{i}\right)$ be the naturally induced coordinates of $T \mathbb{R}^{2 n}$. Then

$$
\widehat{J}:=J_{j}^{i} d x^{j} \otimes \frac{\partial}{\partial x^{i}}+J_{j}^{i} d q^{j} \otimes \frac{\partial}{\partial q^{i}}+q^{k} \frac{\partial J_{j}^{i}}{\partial x^{k}} d x^{j} \otimes \frac{\partial}{\partial q^{i}} .
$$

Then $v$ is a $J$-holomorphic variational vector field along a $J$-holomorphic disc $f$ if and only if $\hat{f}:=(f, v)$ is a $\widehat{J}$-holomorphic disc into the tangent bundle. For more details on complete lifts, one may refer to [11] and to [17].

2. Note that (d) is a special property of the velocity vector field $f^{\prime}$. In general, we cannot produce another $J$-holomorphic variational vector field by multiplying a given $J$-holomorphic variational vector field $v$ by a holomorphic function. 
We have:

Proposition 3.4. Let $f: \Delta \rightarrow \mathbb{R}^{2 n}$ be a $C^{\alpha}$-closed J-holomorphic disc. Let $\mathcal{V}$ be a small neighborhood of $f(\bar{\Delta})$. There exists $\varepsilon>0$ such that if $\left\|J-J_{\mathrm{st}}\right\|_{C^{\alpha-1}(\overline{\mathcal{V}})}<\varepsilon$ then for every $J$-holomorphic variational vector field $v$ along $f$ of class $C^{\alpha-1}$ on $\bar{\Delta}$ and satisfying $v(0)=0$ there exists a one parameter family $h_{t}$ of $C^{\alpha-1}$-closed $J$-holomorphic discs of such that

- $h_{0}=f$,

- $\left.\frac{\mathrm{d}}{\mathrm{d} t}\right|_{t=0} h_{t}=v$,

- $h_{t}^{\prime}(0)=f^{\prime}(0)+t v^{\prime}(0)$.

Proof. We may assume that $f$ is an embedding. Otherwise, consider the $\left(J_{\mathrm{st}} \times J\right)$ holomorphic disc $F(\zeta):=(\zeta, f(\zeta)): \Delta \rightarrow \mathbb{C} \times \mathbb{R}^{2 n}$ and the $\left(J_{\text {st }} \times J\right)$-holomorphic variational vector field $V(\zeta):=(\zeta, v(\zeta))$ along $F$ such that $V(0)=0$.

Let $u_{2}, \ldots, u_{n}$ be $C^{\infty}$ smooth vector fields on a neighborhood of $f(\bar{\Delta})$ such that $f^{\prime}, u_{2}(f), \ldots, u_{n}(f)$ are $\mathbb{C}$-linearly independent for every $\zeta \in \bar{\Delta}$. Consider the $C^{\alpha}$-smooth diffeomorphism

$$
\Phi:\left(\zeta, w^{2}, \ldots, w^{n}\right) \rightarrow f(\zeta)+\sum_{j=2}^{n} w^{j} u_{j}(f(\zeta))
$$

defined on $\Phi^{-1}(\mathcal{V})$, a neighborhood of $f_{0}(\bar{\Delta})$, where $f_{0}(\zeta)=(\zeta, 0, \ldots, 0)$. If we set $J_{\Phi}:=d \Phi^{-1} \circ J \circ d \Phi$ then $\left\|J_{\Phi}-J_{\text {st }}\right\|_{C^{\alpha-1}\left(\overline{\Phi^{-1}(\mathcal{V})}\right)}$ is sufficiently small for an appropriate choice of $u_{2}, \ldots, u_{n}$. Moreover $J_{\Phi}=J_{\text {st }}$ along $f_{0}(\Delta)$. Finally $d \Phi^{-1}(f)(v)$ is a $J_{\Phi}$-holomorphic variational vector field along $f_{0}$ if $v$ is a $J$-holomorphic variational vector field along $f$. We identify $f$ with $f_{0}, J$ with $J_{\Phi}, v$ with $d \Phi^{-1}(f)(v)$ and $\mathcal{V}$ with $\Phi^{-1}(\overline{\mathcal{V}})$ via $\Phi$. Then $J=J_{\text {st }}$ along $f_{0}(\Delta)$.

Let $v$ be a $J$-holomorphic variational vector field along $f_{0}$ with $v(0)=0$, which is $C^{\alpha-1}$ smooth up to $\partial \Delta$. Then $v$ satisfies the equation:

$$
\frac{\partial v}{\partial \bar{\zeta}}+Q v=0
$$

where $\frac{\partial v}{\partial \bar{\zeta}}=\frac{1}{2}\left(\frac{\partial v}{\partial x}+J_{\mathrm{st}} \frac{\partial v}{\partial y}\right)$ and

$$
Q_{j}^{i}(\zeta):=\frac{1}{2} \frac{\partial J_{2}^{i}}{\partial x^{j}}\left(f_{0}(\zeta)\right)
$$

Let

$$
S v:=v+T_{0} Q v
$$


where $T_{0} v(\zeta)=\frac{1}{2 \pi i} \int_{\Delta}\left(\frac{v(\eta)}{\eta-\zeta}-\frac{v(\eta)}{\eta}\right) d \eta \wedge d \bar{\eta}$ is a solution operator for $\partial / \partial \bar{\zeta}$ (see [25]). Then $S$ is an isomorphism of class $C^{\alpha-1}$ on $(\bar{\Delta})$ which is close to the identity map, and $S v$ is a $J_{\text {st }}$-holomorphic map vanishing at $0 \in \Delta$ if and only if $v$ is a $J$-holomorphic variational vector field along $f_{0}$ vanishing at $0 \in \Delta$.

Let $\mathcal{A}$ be the space of triples $(J, v, \phi)$ satisfying:

- $J$ is a $C^{\alpha-1}$-smooth almost complex structure on $\overline{\mathcal{V}}$ such that $J=J_{\text {st }}$ along $f_{0}$,

- $v$ is a $J$-holomorphic variational vector field along $f_{0}$ of class $C^{\alpha-1}$, bounded by 1 , such that $v(0)=0$,

- $\phi:(-\epsilon, \epsilon) \times \bar{\Delta} \rightarrow \mathbb{R}^{2 n}$ is $C^{1}$-smooth, $\phi(t, \cdot)$ is of class $C^{\alpha-1}$ for each $t$ and

$-\phi(0, \cdot)=f_{0}$,

- $\dot{\phi}=v, \phi(t, 0)=0$,

- $\partial \phi / \partial x(t, 0)=\partial f_{0} / \partial x(0)+t \partial v / \partial x(0)$ for every $t$

for some small $\epsilon>0$, where $\dot{\phi}=d \phi /\left.d t\right|_{t=0}$.

Let $G$ be the functional defined on $\mathcal{A}$ by:

$$
G(J, v, \phi)=\bar{\partial}_{J} \phi=\frac{1}{2}\left(\frac{\partial \phi}{\partial x}+J(\phi) \frac{\partial \phi}{\partial y}\right) .
$$

Then the target space of $G$ is:

$\mathcal{B}:=\left\{\psi:(-\epsilon, \epsilon) \times \bar{\Delta} \rightarrow \mathbb{R}^{2 n}: \psi\right.$ of class $C^{1}$ with $\psi(t, \cdot)$ of class $C^{\alpha-2}$ for each $t$, $\psi(0, \cdot)=0, \dot{\psi}=0\}$.

Let $\phi_{0}=f_{0}$. Then

$$
G\left(J_{\text {st }}, 0, \phi_{0}\right)=0 .
$$

The differential $L:=\partial G / \partial \phi$ at $\left(J_{\text {st }}, 0, \phi_{0}\right)$ is

$$
L=\bar{\partial}_{J_{\mathrm{st}}}
$$

from

$\mathcal{C}:=\left\{\xi:(-\epsilon, \epsilon) \times \bar{\Delta} \rightarrow \mathbb{R}^{2 n}: \xi\right.$ of class $C^{1}$ with $\xi(t, \cdot)$ of class $C^{\alpha-1}$ for each $t$,

$$
\begin{aligned}
& \xi(0, \cdot)=\dot{\xi} \equiv 0 \text { and } \\
& \xi(t, 0)=\partial \xi / \partial x(t, 0)=0 \text { for every } t\} .
\end{aligned}
$$

to $\mathcal{B}$. Let $R: \mathcal{B} \rightarrow \mathcal{C}$ be a bounded operator defined by

$$
R \psi(t, \zeta)=T \psi(t, \zeta)-T \psi(t, 0)-\left(\frac{\partial}{\partial \zeta} T \psi(t, 0)\right) \zeta
$$

for every $\psi \in \mathcal{B}$ where

$$
T \psi(t, \zeta)=\frac{1}{2 \pi i} \int_{\Delta} \frac{\psi(t, \eta)}{\eta-\zeta} \mathrm{d} \eta \wedge \mathrm{d} \bar{\eta} .
$$


Then $R$ is a bounded right inverse of $L$. It follows from the Implicit Function Theorem that for every $J$ sufficiently close to $J_{s t}$ in $C^{\alpha-1}$-norm and for every $J$ holomorphic variational vector field $v$ sufficiently small in $C^{\alpha-1}$-norm there is a one parameter family $\phi_{t}$ of class $C^{\alpha-1}$ on $\bar{\Delta}$ satisfying the conclusions of Proposition 3.4. Finally for a given $J$-holomorphic variational vector field $v$ choose $c>0$ such that $c v$ is sufficiently small in $C^{\alpha-1}$ norm. Consider the one parameter family $\phi_{t}$ associated to $c v$ and set $h_{t}:=\phi_{t / c}$.

\section{First order variations}

In this section we give a necessary condition for a $J$-holomorphic disc to be extremal in terms of $J$-holomorphic variational vector fields. Let $\alpha$ be a non-integer real number larger than 2 . We assume that $J$ is a $C^{\alpha}$-smooth almost complex structure on $\mathbb{R}^{2 n}$.

Proposition 4.1. Let $\Omega$ be a bounded domain in $\mathbb{R}^{2 n}$ defined by a $C^{2}$-smooth $J$ plurisubharmonic function $\rho$. Let $f: \Delta \rightarrow \Omega$ be a $C^{\alpha}$-closed extremal disc. Suppose $\left\|J-J_{\mathrm{st}}\right\|_{C^{\alpha-1}}<\varepsilon$ where $\varepsilon$ is given by Proposition 3.4. Then $f$ is attached to the boundary of $\Omega$, that is $f(\partial \Delta) \subset \partial \Omega$.

Proof. Suppose by contradiction that $f$ is not attached to the boundary of $\Omega$. Then there exists an open interval $P$ in $\partial \Delta$ with positive length and a compact subset $K$ of $\Omega$ such that $f(r \zeta) \in K$ for every $\zeta \in P$ and for every $r \leq 1$, sufficiently close to 1 . Choose a relatively compact sub-interval $P_{1}$ in $P$ and let $0 \leq \chi \leq 1$ be a fixed smooth function on $\partial \Delta$, compactly supported in $P$ and equal to one on $P_{1}$. For a positive constant $R$, we denote by $\phi^{R}$ the holomorphic function on $\Delta$ such that $\operatorname{Re} \phi^{R}$ is the Poisson integral of $R \chi$ and $\operatorname{Im} \phi^{R}(0)=0$. For every $r<1$ let $f_{r}(\zeta):=f(r \zeta)$ and let $v_{r}^{R}(\zeta):=\zeta \exp \left(\phi^{R}(\zeta)\right) f_{r}^{\prime}(\zeta)$. Here and throughout the paper we set $f^{\prime}:=\partial f / \partial x$ for every map $f$ defined on an open set of $\mathbb{R}^{2}$. Then $v_{r}^{R}$ is a $J$-holomorphic variational vector field along $f_{r}$. It follows from Proposition 3.4 that there exists a one-parameter family $h_{r, t}^{R}$ of $J$-holomorphic curves defined for $0 \leq t<t_{0}=t_{0}(R)$ such that:

$$
\begin{gathered}
h_{r, 0}^{R}=f_{r}, \\
\left.\frac{\mathrm{d}}{\mathrm{d} t} h_{r, t}^{R}\right|_{t=0}=v_{r}^{R}
\end{gathered}
$$

and

$$
h_{r, t}^{R^{\prime}}(0)=f_{r}^{\prime}(0)+t v_{r}^{R^{\prime}}(0)=r\left(1+t \exp \left(\phi^{R}(0)\right)\right) f^{\prime}(0)
$$

for every $R, r$ and $t$. We point out that $t_{0}$ depends a priori on $r$. So we to take the infimum over $r<1$ close to 1 . Since $\exp \left(\phi^{R}(0)\right) \geq \exp (l R / 2 \pi)$ where $l=$ length of $P_{1}>0$, if $r(1+t \exp (l R / 2 \pi))>1$, or equivalently if

$$
t>t_{1}(r, R):=\frac{1-r}{r} \exp (-l R / 2 \pi)
$$

then $h_{r, t}^{R}$ yields a contradiction to the extremality of $f$ whenever $h_{r, t}^{R}(\Delta) \subset \Omega$. 
Note that for every $J$-holomorphic curve $g: \Delta \rightarrow \Omega$ the function $\rho \circ g$ is subharmonic. So there exists a constant $C_{1}>0$ depending only on $\rho$ and $g(0)$ such that

$$
\rho \circ g(\zeta) \leq-C_{1}(1-|\zeta|)
$$

for every $\zeta \in \Delta$.

Let $\zeta \in \partial \Delta \backslash P$. Then $\left|\exp \left(\phi^{R}(\zeta)\right)\right|=1$. Therefore, $\left|d\left(\rho \circ h_{r, t}^{R}\right) / d t\right|_{t=0} \leq$ $\|d \rho\|_{L^{\infty}(\Omega)}\left\|f^{\prime}\right\|_{L^{\infty}(\Delta)}$. Since the right hand side of this inequality does not depend on $R$, there is a constant $C_{2}>0$ independent of $R$ and $t_{2}(R)>0$ such that

$$
\left|\rho \circ h_{r, t}^{R}(\zeta)-\rho \circ f_{r}(\zeta)\right| \leq C_{2} t
$$

for every $\zeta \in \partial \Delta \backslash P$ and every $0 \leq t<t_{2}(R)$. Therefore, if $C_{2} t \leq C_{1}(1-r)$ or,

$$
t<t_{3}(r):=C_{3}(1-r),
$$

then $h_{r, t}^{R}(\zeta) \in \Omega$ for every $\zeta \in \partial \Delta \backslash P$, where $C_{3}=C_{1} / C_{2}$. We may assume that $t_{3}(r) \leq t_{2}(R)$ since $t_{3}(r) \rightarrow 0$ as $r \rightarrow 1$. Now suppose that $\zeta \in P$. Then there exist positive constants $d=d(K, \rho), C_{4}(R)$ and $t_{4}(R)$ such that

$$
\rho \circ f_{r}(\zeta) \leq-d
$$

and

$$
\left|\rho \circ h_{r, t}^{R}(\zeta)-\rho \circ f_{r}(\zeta)\right|<C_{4}(R) t
$$

for every $t<t_{4}(R)$. Therefore, if $t<\min \left\{t_{4}(R), t_{5}(R)=d / C_{4}(R)\right\}$, then $h_{r, t}^{R}(\zeta) \in \Omega$ for every $\zeta \in P$.

Choose $R$ such that

$$
\exp (-l R / 2 \pi)<C_{3} / 2 .
$$

Then

$$
t_{1}(r, R)<t_{3}(r)
$$

for every $1 / 2<r<1$. Finally, choose $r$ close to 1 so that

$$
t_{3}(r) \leq \min \left\{t_{0}(R), t_{2}(R), t_{4}(R), t_{5}(R)\right\} .
$$

Then for every $t<t_{3}(r), h_{r, t}^{R}$ maps $\Delta$ into $\Omega$ and if we choose $t_{1}(r, R)<t<t_{3}(r)$, then $h_{r, t}^{R}$ yields a contradiction for the extremality of $f$.

Definition 4.2. A $C^{\alpha}$-closed $J$-holomorphic disc $f: \bar{\Delta} \rightarrow \bar{\Omega}$ attached to the boundary of $\Omega$ is said to vanish the first order variations if for every $J$-holomorphic variational vector field $u$ along $f$, of class $C^{\alpha-1}$ on $\bar{\Delta}$, such that:

(a) $\langle\mathrm{d} \rho(f(\zeta)), u(\zeta)\rangle=0$ for every $\zeta \in \partial \Delta$,

(b) $u(0)=0$ and

(c) $\partial u / \partial x(0)=\lambda \partial f / \partial x(0)$ for a $\lambda \in \mathbb{R}$,

it holds that $\lambda=0$. 
We have the following necessary condition for a $J$-holomorphic disc to be extremal:

Theorem 4.3. Let $\Omega$ be a bounded domain in $\mathbb{R}^{2 n}$ defined by a $C^{2}$-smooth $J$ plurisubharmonic function $\rho$. Let $f$ be a $C^{\alpha}$-closed extremal disc in $\Omega$. If $\| J-$ $J_{\mathrm{st}} \|_{C^{\alpha-1}(f(\bar{\Delta}))}<\varepsilon$ for a sufficiently small $\varepsilon>0$, then $f$ vanishes the first order variations.

Proof. We already proved in Proposition 4.1 that $f$ is attached to the boundary of $\Omega$. Suppose that $f$ does not vanish the first order variations. It follows from Definition 4.2 that we may choose a $J$-holomorphic variational vector field $u$ along $f$ such that:

- $\langle\mathrm{d} \rho(f(\zeta)), u(\zeta)\rangle=0$ for every $\zeta \in \partial \Delta$,

- $u(0)=0$,

- $u^{\prime}(0)=\lambda f^{\prime}(0)$ for some $\lambda \in \mathbb{R} \backslash\{0\}$.

We may assume that $\lambda>0$ by taking $-u$, if necessary. Let

$$
v(\zeta):=u(\zeta)-\frac{\lambda}{2} \zeta f^{\prime}(\zeta) .
$$

Then $v$ is also a $J$-holomorphic variational vector field along $f$ of class $C^{\alpha-1}$ on $\bar{\Delta}$ by Proposition 3.2. Choose a 1-parameter family $f_{t}$ for $v$ satisfying the properties of Proposition 3.4. Then for every $\zeta \in \partial \Delta$ :

$$
\begin{aligned}
\left.\frac{\mathrm{d}}{\mathrm{d} t} \rho \circ f_{t}(\zeta)\right|_{t=0} & =\langle\mathrm{d} \rho(f(\zeta)), u(\zeta)\rangle-\frac{\lambda}{2}\left\langle\mathrm{~d} \rho(f(\zeta)), \zeta \frac{\partial f}{\partial x}(\zeta)\right\rangle \\
& =\langle d \rho(f(\zeta)), u(\zeta)\rangle-\left.\frac{\lambda}{2} \frac{\mathrm{d}}{\mathrm{d} r} \rho \circ f(r \zeta)\right|_{r=1} \\
& <0
\end{aligned}
$$

by the hypothesis and by the Hopf lemma. Since $\rho \circ f$ vanishes identically on $\partial \Delta$, then $\rho \circ f_{t}<0$ on $\partial \Delta$ if $t>0$ is small enough. hence $f_{t}$ is a $J$-holomorphic disc into $\Omega$ for every sufficiently small $t>0$. On the other hand,

$$
\begin{aligned}
f_{t}^{\prime}(0) & =f^{\prime}(0)+t v^{\prime}(0) \\
& =\left(1+\frac{\lambda t}{2}\right) f^{\prime}(0) .
\end{aligned}
$$

This yields a contradiction to the extremality of $f$ by choosing small $t>0$.

\section{5. $J$-stationary discs}

Let $\alpha$ be a non integer real number larger than 2. Let $\Omega=\{\rho<0\}$ be a bounded domain in $\mathbb{R}^{2 n}$ where $\rho$ is a $C^{\alpha+1}$-smooth $J$-plurisubharmonic function. Let $J$ be a $C^{\alpha}$-smooth almost complex structure on $\mathbb{R}^{2 n}$ and let $f$ be a $C^{\alpha}$-closed $J$ holomorphic disc in $\Omega$. 


\subsection{Extremal dises are $J$-stationary}

Suppose $f$ is embedded and extremal in $\Omega$. Then $f$ vanishes the first order variations by Theorem 4.3. If $v$ is a $J$-holomorphic variational vector field along $f$, $C^{\alpha}$-smooth on $\bar{\Delta}$, such that $v(0)=0, v^{\prime}(0)=\lambda f^{\prime}(0)$ for some real $\lambda$ and

$$
\langle d \rho(f(\zeta)), v(\zeta)\rangle=0
$$

for every $\zeta \in \partial \Delta$, then it holds that $\lambda=0$.

Let $p$ be a positive function defined for every $\zeta \in \partial \Delta$ by:

$$
\frac{1}{p(\zeta)}=\left.\frac{\mathrm{d}}{\mathrm{d} r} \rho \circ f(r \zeta)\right|_{r=1}=\left\langle\zeta \mathrm{d} \rho(f(\zeta)), f^{\prime}(\zeta)\right\rangle
$$

Let $v$ be a $J$-holomorphic variational vector field along $f, C^{\alpha}$-smooth on $\bar{\Delta}$, and such that $v(0)=v^{\prime}(0)=0$. There is a unique holomorphic function $h$ on $\Delta$, $C^{\alpha}$-smooth on $\bar{\Delta}$, and such that

$$
\operatorname{Re} h=-\left\langle p \mathrm{~d} \rho(f), v_{1}\right\rangle
$$

on $\partial \Delta$ and

$$
\operatorname{Im} h(0)=0 .
$$

Then $v_{1}(\zeta):=v(\zeta)+\zeta h(\zeta) f^{\prime}(\zeta)$ defines a $J$-holomorphic variational vector field along $f, C^{\alpha}$-smooth on $\bar{\Delta}$, which satisfies equation (5.1) and such that $v(0)=0$, $v^{\prime}(0)=h(0) f^{\prime}(0)$. Hence, we see that $f$ vanishes the first order variations if and only if

$$
0=-\lambda=-h(0)=-\operatorname{Re} h(0)=\int_{\partial \Delta}\left\langle p\left(e^{i \theta}\right) \mathrm{d} \rho\left(f\left(e^{i \theta}\right)\right), v\left(e^{i \theta}\right)\right\rangle \frac{\mathrm{d} \theta}{2 \pi}
$$

for every $J$-holomorphic variational vector field $v$ which is smooth on $\bar{\Delta}$, such that $v(0)=v^{\prime}(0)=0$.

Now suppose that $J$ is sufficiently close to $J_{\text {st }}$ in $C^{\alpha}$ norm, in a neighborhood of $f(\bar{\Delta})$. Then every $J$-holomorphic vector field along $f$ which is $C^{\alpha}$-smooth on $\bar{\Delta}$ is completely determined by its boundary values by the classical Schauder theory of linear elliptic partial differential equations. Hence, we may regard the space of $J$-holomorphic vector fields along $f$ which are $C^{\alpha}$-smooth on $\bar{\Delta}$ as a subspace of $C^{\alpha}(\partial \Delta)$. We denote by $\mathcal{A}_{2}$ the $L^{2}(\partial \Delta)$-closure of the space of $J$-holomorphic variational vector field $v$ along $f$ which are $C^{\alpha}$-smooth up to the boundary and such that $v(0)=v^{\prime}(0)=0$. Let

$$
\mathcal{H}_{2}:=\left\{\sum_{k \geq 2} a_{k} e^{i k \theta} \in L^{2}(\partial \Delta): a_{k} \in \mathbb{C}^{n}\right\}
$$


be a subspace of the classical Hardy space. Recall that $v$ is a $J$-holomorphic variational vector field along $f$ if it satisfies:

$$
\frac{\partial v}{\partial x}+J(f) \frac{\partial v}{\partial y}+W v=0
$$

where $W=\left(W_{j}^{i}\right)$ is given by

$$
W_{j}^{i}=\frac{\partial J_{k}^{i}}{\partial x^{j}}(f) \frac{\partial f^{k}}{\partial y}
$$

for every $i, j=1, \ldots, 2 n$. Let

$$
\tilde{S} v=\tilde{S}_{J, f} v:=v+K v-K v(0)-\left\{\frac{\partial}{\partial \zeta} K v(0)\right\} \zeta
$$

for $v \in C^{\alpha}(\bar{\Delta})$, where $K=K_{J, f}$ is a linear operator defined by

$$
K v:=\frac{1}{2} T\left\{\left(J(f)-J_{\mathrm{st}}\right) \frac{\partial}{\partial y}+W\right\} v
$$

and $T$ is the Cauchy-Green operator defined by

$$
T u(\zeta)=\frac{1}{2 \pi i} \int_{\Delta} \frac{u(\eta)}{\eta-\zeta} d \eta \wedge d \bar{\eta} .
$$

Fix a $C^{\alpha}$-closed $J_{\mathrm{st}}$-holomorphic disc $f_{0}$. Then $\tilde{S}$ is a bounded operator on $C^{\alpha}(\bar{\Delta})$ to itself, which tends to the identity operator as $f$ converges to $f_{0}$ in $C^{\alpha}$ norm on $\bar{\Delta}$ and $J$ converges to $J_{\text {st }}$ in $C^{\alpha}$ norm on a neighborhood of $f_{0}(\bar{\Delta})$. Moreover, $v$ is a $J$-holomorphic variational vector field along $f$ satisfying $v(0)=0$ and $v^{\prime}(0)=0$ if and only if $\tilde{S} v$ is $J_{\mathrm{st}}$-holomorphic and satisfies $\tilde{S} v(0)=0$ and $(\tilde{S} v)^{\prime}(0)=0$. Therefore, $\mathcal{A}_{2} \cap C^{\alpha}(\bar{\Delta})$ is isomorphic to $\mathcal{H}_{2} \cap C^{\alpha}(\bar{\Delta})$ and they are close to each other in $C^{\alpha}$-sense. This implies that $\mathcal{A}_{2}$ is isomorphic to $\mathcal{H}_{2}$, and is a small perturbation of $\mathcal{H}_{2}$ in $L^{2}(\partial \Delta)$. Moreover since $T$ is a bounded operator from $C^{\alpha-1}(\bar{\Delta})$ to $C^{\alpha}(\bar{\Delta})$ then for a fixed $v$, the correspondence $(J, f) \rightarrow \tilde{S}_{J, f} v$ is $C^{1}$-smooth. This implies that $\mathcal{A}_{2} \cap C^{\alpha}(\bar{\Delta})$ varies $C^{1}$-smoothly as $(J, f)$ changes in $C^{\alpha}$-space.

Since $\mathcal{A}_{2}$ is sufficiently close to $\mathcal{H}_{2}$ in $L^{2}(\partial \Delta)$, the $L^{2}$-projection $\pi$ : $L^{2}(\partial \Delta) \rightarrow \mathcal{H}_{2}$ induces an isomorphism from $\mathcal{A}_{2}$ onto $\mathcal{H}_{2}$. Let $\mathcal{H}_{2}^{\perp}$ be the orthogonal complement of $\mathcal{H}_{2}$ in $L^{2}(\partial \Delta)$ and let $\Pi$ be the isomorphism of $L^{2}(\partial \Delta)$ onto itself defined by

$$
\Pi(v):= \begin{cases}v & \text { if } v \in \mathcal{H}_{2}^{\perp} \\ \pi(v) & \text { if } v \in \mathcal{A}_{2} .\end{cases}
$$


If $f$ vanishes the first order variations, then from equation (5.2) we have:

$$
\begin{aligned}
0 & =\int_{\partial \Delta}\langle p \mathrm{~d} \rho(f), v\rangle \mathrm{d} \theta \\
& =\int_{\partial \Delta}\left\langle\left(\Pi^{-1}\right)^{*}(p \mathrm{~d} \rho(f)), \Pi(v)\right\rangle \mathrm{d} \theta
\end{aligned}
$$

for every $v \in \mathcal{A}_{2}$, where $\left(\Pi^{-1}\right)^{*}$ is the $L^{2}$-adjoint of $\Pi^{-1}$. By the definition of $\Pi$, $\Pi(v) / \zeta^{2}$ is an arbitrary standard holomorphic map contained in $L^{2}(\partial \Delta)$. Therefore, $f$ vanishes the first order variations if

$$
\int_{\partial \Delta}\left\langle\zeta\left(\Pi^{-1}\right)^{*}(p \mathrm{~d} \rho(f)), h\right\rangle \mathrm{d} \zeta=0
$$

for every standard holomorphic map $h \in L^{2}(\partial \Delta)$. This implies that the correspondence $\partial \Delta \ni \zeta \rightarrow \zeta\left(\Pi^{-1}\right)^{*}(p \mathrm{~d} \rho(f))(\zeta)$ extends continuously to a $J_{\mathrm{st}^{-}}^{t}$ holomorphic mapping defined on $\Delta$. Following a viewpoint of [14], we define $J$-stationary discs for a structure $J$ sufficiently close to $J_{\text {st }}$ as follows:

Definition 5.1. Let $\alpha>1$, non integer. Let $\Omega=\{\rho<0\}$ be a bounded domain with $\rho$ of class $C^{\alpha+1}$. Let $f$ be a properly embedded $C^{\alpha}$-closed $J$-holomorphic disc. Suppose $J$ is sufficiently close to $J_{\text {st }}$ in a neighborhood of $f(\bar{\Delta})$ in $C^{\alpha}$ norm so that the isomorphism $\Pi$ in (5.3) is well-defined. Then we say that $f$ is $J$-stationary if there exists a positive $C^{\alpha}$-smooth map $p$ defined on $\partial \Delta$ such that the correspondence $\partial \Delta \ni \zeta \rightarrow \zeta\left(\Pi^{-1}\right)^{*}(p d \rho(f))(\zeta)$ continuously extends to a $J_{\mathrm{st}}^{t}$-holomorphic mapping defined on $\Delta$. Here, the multiplication by $\zeta$ to 1 -forms is defined by $J_{\mathrm{st}}^{t}$.

It should be mentioned that the positive function $p$ in Definition 5.1 may be modified by multiplying a given one by real positive constants. Hence we may assume that $p(1)=1$.

We have proved the following theorem:

Theorem 5.2. Let $J$ be a $C^{\infty}$-smooth almost complex structure on $\mathbb{R}^{2 n}$ and let $\Omega$ be a bounded domain with a $C^{\infty}$-smooth J-plurisubharmonic defining function $\rho$. Let $f$ be a $C^{\infty}$-closed extremal disc embedded into $\Omega$. Suppose that $J$ is sufficiently close to $J_{\text {st }}$ in a neighborhood of $f(\bar{\Delta})$ in $C^{\alpha}$ norm $(\alpha>1$, non-integer). Then $f$ is $J$-stationary.

Proof. According to Theorem $4.3 f$ vanishes the first order variations. It follows from the discussion preceding Theorem 5.2 that $f$ is $J$-stationary.

\subsection{Existence of $J$-stationary discs}

If $J=J_{\text {st }}$ and $D$ is a strongly convex domain in $\mathbb{C}^{n}$, it is known by [14] that there exists a unique $J_{\mathrm{st}}$-stationary disc $f_{0}$ such that $f_{0}(0)=0$ and $f^{\prime}(0)=\lambda_{0} V$ for some $\lambda_{0}>0$. We prove the following: 
Theorem 5.3. Let $\alpha>1$, not integer. Let $\Omega=\{\rho<0\}$ be a bounded strongly convex domain in $\mathbb{R}^{2 n}$, where $\rho$ is $C^{\alpha+3}$-smooth. Let $z_{0} \in \Omega$. Then there exists $\epsilon>0$ such that if an almost complex structure $J$ on $\mathbb{R}^{2 n}$ satisfies $\left|J-J_{\mathrm{st}}\right|_{C^{\alpha}(\bar{\Omega})}<\epsilon$, then for every nonzero vector $V$ in $\mathbb{R}^{2 n}$, there exists a unique $J$-stationary disc $f$ which is $C^{\alpha}$-smooth on $\bar{\Delta}$, with $f(0)=z_{0}$ and $f^{\prime}(0)=\lambda V$ for some $\lambda>0$.

Proof. We may assume without loss of generality that $z_{0}=0 \in \Omega$. Let $V$ be a nonzero vector in $\mathbb{R}^{2 n}$. Let $X$ be defined by:

$$
\begin{aligned}
X:=\{(J, f): & J \text { is almost complex structure on } \mathbb{R}^{2 n}, \\
& \left.f \text { is } C^{\alpha} \text {-smooth closed } J \text {-holomorphic disc such that } f(0)=0\right\},
\end{aligned}
$$

endowed with the $C^{\alpha}$ topology. Then $X$ is a Banach manifold defined in a neighborhood of $\left(J_{\mathrm{st}}, f_{0}\right)$. Denote by $P$ the space of $C^{\alpha}$-smooth positive functions on $\partial \Delta$, equal to 1 at point 1 , and by $\mathbb{R}^{+}$the space of non negative real numbers. Consider a functional $F$ from $X \times P \times \mathbb{R}^{+}$to $C^{\alpha}(\partial \Delta) \times \mathbb{R}^{2 n} \times H^{\alpha}$ defined by:

$$
G(J, f, p, \lambda):=\left(\left.\rho \circ f\right|_{\partial \Delta}, f^{\prime}(0)-\lambda V, \pi_{1}\left(\bar{\zeta}\left(\Pi^{-1}\right)^{*}(p D \rho(f))\right)\right.
$$

where $\pi_{1}: L^{2}(\partial \Delta) \rightarrow L^{2}(\partial \Delta)$ is the $L^{2}$-projection defined by

$$
\begin{gathered}
\pi_{1}\left(\sum_{-\infty}^{\infty} a_{k} e^{i k \theta}\right)=\sum_{k=1}^{\infty} a_{k} e^{i k \theta}, \\
D \rho=\left(\frac{\partial \rho}{\partial x^{1}}, \ldots, \frac{\partial \rho}{\partial x^{2 n}}\right)
\end{gathered}
$$

and $H^{\alpha}$ is the space of the standard holomorphic mappings into $\mathbb{C}^{n}$ which are $C^{\alpha}$ smooth on $\bar{\Delta}$ and vanish at the origin.

Then $G$ is a $C^{1}$-smooth functional, since the space $\mathcal{A}_{2}$ varies $C^{1}$ smoothly when $J$ and $f$ change in $X$. Note that for a 1 -form $u=\sum u_{j} d x^{j}, \zeta u$ is $J_{\text {st }^{-}}$ holomorphic if and only if the map $\bar{\zeta}\left(u_{1}, \ldots, u_{2 n}\right)$ with values in $\mathbb{C}^{n}=\left(\mathbb{R}^{2 n}, J_{\mathrm{st}}\right)$ is anti-holomorphic. Therefore:

A $J$-holomorphic disc $f$ such that $f(0)=0$ and $f^{\prime}(0)=\lambda V$ is $J$-stationary if and only if there exists $p \in P$ such that $G(J, f, p, \lambda)=0$.

Since $G\left(J_{\mathrm{st}}, f_{0}, p_{0}, \lambda_{0}\right)=0$, it suffices to show that

$$
L:=\frac{\partial G}{\partial(f, p, \lambda)}\left(J_{\mathrm{st}}, f_{0}, p_{0}, \lambda_{0}\right)
$$

is an isomorphism from

$Y:=\left\{(F, Q, \Lambda): F\right.$ is holomorphic on $\Delta$ which is $C^{\alpha}$-smooth on $\bar{\Delta}, F(0)=0$, $Q$ is a $C^{\alpha}$-smooth real valued function on $\partial \Delta, Q(1)=0$, $\Lambda \in \mathbb{R}\}$ 
onto $Z:=C^{\alpha}(\partial \Delta) \times \mathbb{R}^{2 n} \times H^{\alpha}$, where $p_{0}: \partial \Delta \rightarrow \mathbb{R}^{+}$is defined by

$$
p_{0}(\zeta)=\left(\left.\frac{\partial}{\partial r}\right|_{r=1} \rho\left(f_{0}(r)\right)\right)\left(\left.\frac{\partial}{\partial r}\right|_{r=1} \rho\left(f_{0}(r \zeta)\right)\right)^{-1}
$$

We point out that since $f_{0}$ is a stationary disc with respect to $J_{\text {st }}$, it is $C^{\alpha+1}$-smooth if $\rho$ is $C^{\alpha+3}$-smooth by [14]. Therefore, $p_{0}$ is $C^{\alpha}$-smooth.

Since $\Pi$ is the identity if $J=J_{\mathrm{st}}, L$ is equal to the differential of the functional defined to prove the existence of stationary discs; this is known to be invertible by the results of L. Lempert [15] and M. Pang [19]. For the sake of completeness, we state below a sketch of proof for the invertibility of $L$.

We first mention that there exists a biholomorphic change defined in a neighborhood $f_{0}(\bar{\Delta})$ which yields that

- $f_{0}(\zeta)=(\zeta, 0, \ldots, 0)$

- $\rho(\zeta, w)=-1+|\zeta|^{2}+|w|^{2}+\operatorname{Re} B_{\mu \nu}(\zeta) w^{\mu} w^{v}+r(\zeta, w)$

where $r(\zeta, w)=O\left(|w|^{3}\right)$. Such a pair $\left(f_{0}, \rho\right)$ is said to be in normal form (see [19].) Under this normalization, $p_{0} \equiv 1$ and we may assume that $V=e_{1}=$ $(1,0, \ldots, 0)$ and $\lambda_{0}=1$.

In complex notations,

$$
D \rho=\partial \rho / \partial \bar{\zeta}=2\left(\partial \rho / \partial \bar{z}_{1}, \ldots, \partial \rho / \partial \bar{z}_{n}\right) .
$$

To prove the invertibility of $L$, we have to prove that the following system of equations (5.4), (5.5) and (5.6) has a unique solution $(F, Q, \Lambda) \in Y$

$$
\begin{gathered}
\operatorname{Re}\left(\frac{\partial \rho}{\partial z^{\mu}}\left(f_{0}\right)\right) F^{\mu}=r, \\
F^{\prime}(0)-\Lambda e_{1}=U,
\end{gathered}
$$

and

$$
\bar{\pi}_{1}\left\{\zeta Q \frac{\partial \rho}{\partial z^{\mu}}\left(f_{0}\right)+\zeta \frac{\partial^{2} \rho}{\partial z^{\mu} \partial z^{\bar{v}}}\left(f_{0}\right) F^{\bar{v}}+\zeta \frac{\partial^{2} \rho}{\partial z^{\mu} \partial z^{\nu}}\left(f_{0}\right) F^{\nu}\right\}=\overline{h^{\mu}}
$$

for a given $(r, U, h) \in Z$, where $\bar{\pi}_{1}(\bar{g}):=\overline{\pi_{1}(g)}, F=\left(F^{1}, \ldots, F^{n}\right)$ and $h=$ $\left(h^{1}, \ldots, h^{n}\right)$ in complex coordinates. Let

$$
F(\zeta)=\zeta \phi^{\mu}(\zeta) e_{\mu}
$$

for some holomorphic functions $\phi^{1}, \ldots, \phi^{n}$ which are $C^{\alpha}$-smooth on $\bar{\Delta}$, where $e_{1}, \ldots, e_{n}$ is the standard basis for the complex Euclidean space $\mathbb{C}^{n}$. From (5.4) we get

$$
\operatorname{Re} \phi^{1}=r
$$


on $\partial \Delta$. Since $F^{\prime}(0)=\phi^{\mu}(0) e_{\mu}$, we have

$$
\phi^{1}(0)=\Lambda+U^{1}
$$

and

$$
\phi^{\mu}(0)=U^{\mu}
$$

for $\mu=2, \ldots, n$. Since $\phi^{1}$ is determined uniquely up to imaginary constant by (5.8) and $\operatorname{Im} \phi^{1}(0)=\operatorname{Im} U^{1}$ by (5.9), $\phi^{1}$ and $\Lambda$ are determined uniquely. The equation (5.6) implies that

$$
Q+\zeta \overline{F^{1}}=\overline{h^{1}}+g
$$

and

$$
\zeta \overline{F^{\mu}}+\zeta \sum_{\nu \geq 2} B_{\mu \nu} F^{v}=\overline{h^{\mu}}+g^{\mu}
$$

if $\mu=2, \ldots, n$, for some holomorphic functions $g$ and $g^{\mu}$ which are $C^{\alpha}$-smooth on $\bar{\Delta}$. From (5.11), the negative Fourier coefficients of $Q$ are uniquely determined. Moreover, since $Q(1)=0$ and $Q$ is a real-valued function, $Q$ is completely determined.

To determine $F^{\mu}=\zeta \phi^{\mu}$, we introduce a lemma on the unique solvability of a linear problem proved by L. Lempert [15].

Lemma 5.4. Let $\beta$ be a $C^{\alpha}$-smooth $m \times m$ symmetric matrix-valued function on $\partial \Delta$. Suppose that

$$
|w|^{2}>\left|\beta_{\mu \nu}(\zeta) w^{\mu} w^{v}\right|
$$

for every $\zeta \in \partial \Delta$ and $w \in \mathbb{C}^{m} \backslash\{0\}$. Let $h: \partial \Delta \rightarrow \mathbb{C}^{m}$ be a $C^{\alpha}$-smooth map and $a \in \mathbb{C}^{m}$. Then there exists a unique holomorphic map $\phi: \Delta \rightarrow \mathbb{C}^{m}$ which is $C^{\alpha}$-smooth on $\bar{\Delta}$ such that $\phi(0)=a$ and $\bar{\phi}+\beta \phi+h$ extends to a holomorphic map on $\Delta$.

L. Lempert [15] proved this lemma when $0<\alpha<1$. It can be seen that it is still valid for every non-integral real $\alpha>1$.

We complete the proof of the invertibility of $L$ by using Lemma 5.4. From equation (5.7) and (5.12),

$$
\bar{\phi}+\beta \phi-\bar{h}=g
$$

where $\phi=\left(\phi^{2}, \ldots, \phi^{n}\right), h=\left(h^{2}, \ldots, h^{n}\right), g=\left(g^{2}, \ldots, g^{n}\right)$ and $\beta_{\mu \nu}=\zeta^{2} B_{\mu \nu}$. The matrix valued function $\beta$ satisfies the inequality (5.13) because of the strong convexity of $\Omega$. Since $g$ is holomorphic, $\phi$ and hence $F$ are determined uniquely by (5.10). 


\section{Second order variations}

Let $\Omega=\{\rho<0\}$ be a bounded domain in $\mathbb{R}^{2 n}$ with $\rho$ being $C^{\infty}$-smooth. Let $J$ be a $C^{\infty}$-smooth almost complex structure on $\mathbb{R}^{2 n}$. Assume that $f$ is an $J$-stationary disc which is $C^{\infty}$-smooth up to the boundary. We assume that $J$ is close to $J_{\text {st }}$ in a neighborhood of $f(\bar{\Delta})$ so that $\Pi$ is a well-defined isomorphism.

Let $\mathcal{F}$ be the space of $C^{\infty}$-closed $J$-holomorphic discs into $\mathbb{R}^{2 n}$ such that if $g \in \mathcal{F}$, then $g(0)=f(0)$ and $g^{\prime}(0)=f^{\prime}(0)$. Let $p \in P$ be a positive function for $f$ on $\partial \Delta$ given in Definition 5.1. Let $F$ be a functional defined on $\mathcal{F}$ by

$$
F(g):=\int_{\partial \Delta} p\left(e^{i \theta}\right) \rho\left(g\left(e^{i \theta}\right)\right) d \theta
$$

where $\rho$ is a defining function of $\Omega$. Let $g_{t}$ be a smooth 1-parameter family in $\mathcal{F}$ such that $g_{0}=f$. Then

$$
\begin{aligned}
\left.\frac{\mathrm{d}}{\mathrm{d} t} F\left(g_{t}\right)\right|_{t=0} & =\int_{\partial \Delta}\langle p \mathrm{~d} \rho(f), \dot{g}\rangle \mathrm{d} \theta \\
& =\int_{\partial \Delta}\left\langle\zeta\left(\Pi^{-1}\right)^{*}(p \mathrm{~d} \rho(f)), \frac{\Pi \dot{g}}{\zeta}\right\rangle \mathrm{d} \theta \\
& =0
\end{aligned}
$$

since $f$ is $J$-stationary and $\frac{\Pi \dot{g}}{\zeta}$ is a holomorphic map which vanishes at 0 , where $\dot{g}=\mathrm{d} g_{t} /\left.\mathrm{d} t\right|_{t=0}$. This implies that $f$ is a critical point of $F$.

Now we think of the second order differentiation of $F\left(g_{t}\right)$. We first mention that a J-holomorphic disc which is $C^{\infty}$-smooth up to $\partial \Delta$ is completely determined by its values on $\partial \Delta$. This is a consequence of the unique continuation principle (see the more general result [12]). Let $g_{1}$ and $g_{2}$ be two $J$-holomorphic discs which are smooth on $\bar{\Delta}$. Suppose that $g_{1}=g_{2}$ on an open arc in $\partial \Delta$. By the Caley transform, we may assume that $g_{1}$ and $g_{2}$ are defined on the upper half plane $U=$ $\{\zeta \in \mathbb{C}: \operatorname{Im} \zeta>0\}$ which are smooth up to the real axis $\mathbb{R}$ and that $g_{1}=g_{2}$ on an open interval $I$ in $\mathbb{R}$. Let $h:=g_{1}-g_{2}$. Obviously, $h \equiv 0$ and $\partial h / \partial x \equiv 0$ on $I$. Moreover, since $\partial g_{1} / \partial y-J\left(g_{1}\right) \partial g_{1} / \partial x=0$ and $\partial g_{2} / \partial y-J\left(g_{2}\right) \partial g_{2} / \partial x=0$, $\partial h / \partial y \equiv 0$ on $I$, too. Differentiating successively Cauchy-Riemann equations for $g_{1}$ and $g_{2}$, it can be seen that every higher order derivative of $h$ vanishes identically on $I$. Fix a point $p \in I$ and choose a small $\epsilon$-disc $B_{\epsilon}$ in $\mathbb{C}$ centered at $p$. Define a mapping $\tilde{h}$ on $B_{\epsilon}$ by

$$
\tilde{h}(\zeta)= \begin{cases}h(\zeta) & \text { if } \operatorname{Im} \zeta \geq 0 \\ h(\bar{\zeta}) & \text { if } \operatorname{Im} \zeta<0\end{cases}
$$

Then, $\tilde{h}$ is a $C^{\infty}$-smooth mapping which vanishes at $p$ up to infinite order. Consequently, $\tilde{h}$ vanishes identically on $B_{\epsilon}$ by the following lemma and this implies that $g_{1} \equiv g_{2}$ on $U$ by the standard unique continuation principle. 
Lemma 6.1 (See [1,10]). A smooth function $u$ on $B_{\epsilon}$ which vanishes at $p$ up to infinite order and which satisfy

$$
\left|\nabla^{2} u\right| \leq C(|u|+|\partial u / \partial x|+|\partial u / \partial y|)
$$

on $B_{\epsilon}$ for some positive constant $C$ vanishes identically on $B_{\epsilon}$.

Hence, we may regard $\mathcal{F}$ as a subspace of $L^{2}(\partial \Delta)$. Let $\overline{\mathcal{F}}$ the $L^{2}$-closure of $\mathcal{F}$ in $L^{2}(\partial \Delta)$. Then $\overline{\mathcal{F}}$ becomes a Hilbert submanifold of $L^{2}(\partial \Delta)$ and

$$
T_{f} \overline{\mathcal{F}}=\mathcal{A}_{2}
$$

We denote by $\ddot{g}$ the second order differentiation of $g_{t}$ at $t=0$. Let $\ddot{g}^{T}$ be the orthogonal projection of $\ddot{g}$ to $\mathcal{A}_{2}$. Then

$$
\ddot{g}=\ddot{g}^{T}+\mathcal{L}_{J}(\dot{g}, \dot{g})
$$

where $\mathcal{L}_{J}: \mathcal{A}_{2} \times \mathcal{A}_{2} \rightarrow L^{2}(\partial \Delta)$ is a symmetric bilinear form, which is called the second fundamental form of the Hilbert submanifold $\overline{\mathcal{F}}$ at $f$. Without loss of generality we may assume that $f=f_{0}=(\zeta, 0, \cdots, 0)$ and $J=J_{\text {st }}$ alonf $f_{0}(\Delta)$.

We claim that:

$$
\left\|\left|\mathcal{L}_{J} \|\right| \rightarrow 0 \text { as }\right\| J-J_{\text {st }} \|_{C^{\alpha}} \rightarrow 0
$$

where $\left\|\mid \mathcal{L}_{J}\right\|$ denotes the operator norm of $\mathcal{L}_{J}$.

Indeed for $g \in C^{\alpha}(\bar{\Delta})$ consider $\widehat{S}_{J} g(\zeta)=g(\zeta)+T\left(J(g)-J_{\mathrm{st}}\right) \frac{\partial g}{\partial y}-T(J(g)-$ $\left.J_{\mathrm{st}}\right) \frac{\partial g}{\partial y}(0)-\frac{\partial}{\partial \zeta}\left(T\left(J(g)-J_{\mathrm{st}}\right) \frac{\partial g}{\partial y}\right)(0) \zeta$.

Then $g$ is a $J$-holomorphic disc of class $C^{\alpha}$ on $\bar{\Delta}$ satisfying $g(0)=0$ and $g^{\prime}(0)=f^{\prime}(0)$ if and only if $\widehat{S}_{J} g$ is a $J_{\text {st }}$-holomorphic disc of class $C^{\alpha}$ on $\bar{\Delta}$ satisfying $\widehat{S}_{J} g(0)=0$ and $\left(\widehat{S}_{J} g\right)^{\prime}(0)=f^{\prime}(0)$.

Moreover for a fixed $g$ the map $J \mapsto \widehat{S}_{J} g$ is $C^{2}$-smooth from the set of almost complex structures on $\mathbb{R}^{2 n}$ endowed with the $C^{\alpha}$-topology to the $L^{2}(\partial \Delta)$. Hence $\overline{\mathcal{F}}$ is a $C^{2}$-smooth deformation of an affine space consisting of $J_{\mathrm{st}}$-holomorphic maps. This implies that the map $J \mapsto \mathcal{L}_{J}$ is at least continuous. The conclusion comes from $\mathcal{L}_{J_{\text {st }}} \equiv 0$.

Now we compute the second order differentiation of $F\left(g_{t}\right)$ :

$$
\begin{aligned}
\left.\frac{\mathrm{d}^{2}}{\mathrm{~d} t^{2}} F\left(g_{t}\right)\right|_{t=0}= & \int_{\partial \Delta} p \frac{\partial^{2} \rho}{\partial x^{j} x^{k}}(f) \dot{g}^{j} \dot{g}^{k} \mathrm{~d} \theta+\int_{\partial \Delta} p\langle d \rho(f), \ddot{g}\rangle \mathrm{d} \theta \\
= & \int_{\partial \Delta} p \frac{\partial^{2} \rho}{\partial x^{j} x^{k}}(f) \dot{g}^{j} \dot{g}^{k} d \theta+\int_{\partial \Delta} p\left\langle d \rho(f), \ddot{g}^{T}\right\rangle \mathrm{d} \theta \\
& +\int_{\partial \Delta} p\left\langle d \rho(f), \mathcal{L}_{J}(\dot{g}, \dot{g})\right\rangle \mathrm{d} \theta \\
= & \int_{\partial \Delta} p \frac{\partial^{2} \rho}{\partial x^{j} x^{k}}(f) \dot{g}^{j} \dot{g}^{k} \mathrm{~d} \theta+\int_{\partial \Delta} p\left\langle d \rho(f), \mathcal{L}_{J}(\dot{g}, \dot{g})\right\rangle \mathrm{d} \theta
\end{aligned}
$$


The second equality comes from (6.2) and the last equality is a consequence of the fact that $f$ is a critical point of $F$ on $\mathcal{F}$. Therefore, if the real Hessian of $\rho$ along $f(\partial \Delta)$ is positive definite and $J$ is sufficiently close to $J_{\text {st }}$, it is deduced from (6.3) that

$$
\left.\frac{\mathrm{d}^{2}}{\mathrm{~d} t^{2}} F\left(g_{t}\right)\right|_{t=0}>0 .
$$

Definition 6.2. Let $f$ be a $J$-holomorphic disc properly embedded in $\{\rho<0\}$. The defining function $\rho$ is strongly convex along $f(\partial \Delta)$ if the real Hessian $\left(\frac{\partial^{2} \rho}{\partial x^{j} \partial x^{k}}\right)$ $(f(\zeta))$ is positive definite for every $\zeta \in \partial \Delta$.

Definition 6.3. A $J$-holomorphic disc $f: \Delta \rightarrow \Omega$ is called a locally extremal disc if there exists $\epsilon>0$ such that for every $J$-holomorphic disc $g: \Delta \rightarrow \Omega$ in an $\epsilon$ neighborhood of $f$ in $L^{\infty}(\Delta)$ which satisfies that $g(0)=f(0)$ and $g^{\prime}(0)=\lambda f^{\prime}(0)$ for some positive real number $\lambda$, it holds that $\lambda \leq 1$.

Theorem 6.4. Let $\Omega=\{\rho<0\}$ be a bounded domain in $\mathbb{R}^{2 n}$ defined by a $C^{\infty}$ smooth function $\rho$ and let $J$ be a $C^{\infty}$-smooth almost complex structure on $\mathbb{R}^{2 n}$. Suppose that $f: \Delta \rightarrow \Omega$ is a $C^{\infty}$-closed J-holomorphic disc which is $J$-stationary. Assume that $J$ is sufficiently close to $J_{\text {st }}$ in a neighborhood of $f(\bar{\Delta})$ in $C^{\alpha}$-sense for some $\alpha>2$ and that $\rho$ is strongly convex along $f(\partial \Delta)$. Then $f$ is a locally extremal disc.

Proof. Assume by contradiction that $f$ is not locally extremal. Then there exists a real number $\lambda>1$ which is close to 1 and there exists a $J$-holomorphic disc $g: \Delta \rightarrow \Omega$ which is close to $f$ in $L^{\infty}(\Delta)$ norm such that $g(0)=f(0)$ and $g^{\prime}(0)=\lambda f^{\prime}(0)$. Let $g_{\lambda}(\zeta):=g(\zeta / \lambda)$. Then $g_{\lambda}$ is a $C^{\infty}$-closed $J$-holomorphic disc belonging to $\mathcal{F}$, which is close to $f$ in $L^{\infty}(\Delta)$ norm. Since the functional $F$ defined by (6.1) is twice differentiable on the space of closed $J$-holomorphic discs endowed with $L^{\infty}$-topology because $\rho$ is $C^{\infty}$-smooth, (6.4) means that $f$ is a local minimum of $F$ on $\mathcal{F}$. In particular if $h \in \mathcal{F}$ is close to $f$ in $L^{\infty}(\Delta)$ norm and does not coincide with $f$, then the image of $h$ is not included in $\Omega$. Therefore, $g_{\lambda}=f$, since $g_{\lambda}$ maps $\Delta$ into $\Omega$. However, since $\rho \circ g$ is a subharmonic function on $\Delta$ which is strictly less than 0 , there exists a positive constant $C$ such that

$$
\rho \circ g(\zeta) \leq-C(1-|\zeta|)
$$

for every $\zeta \in \Delta$. This implies that

$$
\rho \circ g_{\lambda}(\zeta) \leq-C\left(1-\frac{1}{\lambda}\right)<0
$$

for every $\zeta \in \partial \Delta$. Therefore, $g_{\lambda}(\Delta)$ is relatively compact in $\Omega$, which contradicts the fact that $f$ is attached to $\partial \Omega$. 


\section{References}

[1] N. Aronszajn, A unique continuation theorem for solutions of elliptic partial differential equations or inequalities of second order, J. Math. Pures Appl. (9) 36 (1957), 235-249.

[2] M. Audin and J. Lafontaine, "Holomorphic Curves in Symplectic Geometry", Progr. Math., Vol. 117, Birkhäuser, Basel, 1994.

[3] J. BLAND and T. DUChAMP, Moduli for pointed convex domains, Invent. Math. 104 (1991), 61-112.

[4] D. BURNS and S. KRANTZ, Rigidity of holomorphic mappings and a new Schwarz lemma at the boundary, J. Amer. Math. Soc. 7 (1994), 661-676.

[5] Y. J. CHOE, The Kobayashi geometry of Lee's model of dimension 6, preprint.

[6] B. Coupet, H. GAussier and A. SuKHOV, Fefferman's mapping theorem on almost complex manifolds in complex dimension two, Math. Z. 250 (2005), 59-90.

[7] B. Coupet, H. GAUSSIER and A. SuKHOv, Riemann maps in almost complex manifolds, Ann. Scuola Norm. Super. Pisa Cl. Sci. (5) 2 (2003), 761-785.

[8] H. GAUSSIER and A. SUKHOV, On the geometry of model almost complex manifolds with boundary, Math. Z. 254 (2006), 567-589.

[9] M. Gromov, Pseudo holomorphic curves in symplectic manifolds, Invent. Math. 82 (1985), 307-347.

[10] P. HARTMAN and A. WINTNER, On the local behavior of solutions of non-parabolic partial differential equations, Amer. J. Math. 75 (1953), 449-476.

[11] S. Ishihara and K. YAno, "Tangent and Cotangent Bundles: Differential Geometry", Pure and Applied Mathematics, No. 16, Marcel Dekker, Inc., New York, 1973.

[12] S. IVAShKovich and J. P. RosAY, Boundary Values and Boundary Uniqueness of JHolomorphic Mappings, arXiv:0902.4800.

[13] K.-H. LEE, Strongly pseudoconvex homogeneous domains in almost complex manifolds, J. Reine Angew. Math. 623 (2008), 123-160.

[14] L. LEMPERT, La métrique de Kobayashi et la représentation des domaines sur la boule, (French) [The Kobayashi metric and the representation of domains on the ball] Bull. Soc. Math. France 109 (1981), 427-474.

[15] L. LEMPERT, A precise result on the boundary regularity of biholomorphic mappings, Math. Z. 193 (1986), 559-579.

[16] L. LEMPERT, Holomorphic invariants, normal forms, and the moduli space of convex domains, Ann. of Math. 128 (1988), 43-78.

[17] L. LEMPERT and R. SzÖKE, The tangent bundle of an almost complex manifold, Canad. Math. Bull. 44 (2001), 70-79.

[18] D. McDuff and D. Salamon, “ J-holomorphic Curves and Symplectic Topology", Amer. Math. Soc., Coll. Publ., Vol. 52, 2004.

[19] M. -Y. PANG, Smoothness of the Kobayashi metric of nonconvex domains, Internat. J. Math. 4 (1993), 953-987.

[20] G. Patrizio, Real and complex Monge Ampère equations and the geometry of strictly convex domains, Publ. Res. Inst. Math. Sci. 20 (1984), 867-875.

[21] E. A. PoletsKy, The Euler Lagrange equations for extremal holomorphic mappings of the unit disk, Michigan Math. J. 30 (1983), 317-333.

[22] A. SPIRO, Total reality of conormal bundles of hypersurfaces in almost complex manifolds, Int. J. Geom. Methods Mod. Phys. 3 (2006), 1255-1262.

[23] A. SPIRO and A. SuKhov, An existence theorem for stationary discs in almost complex manifolds, J. Math. Anal. Appl. 327 (2007), 269-286.

[24] A. Tumanov, Extremal discs and the regularity of CR mappings in higher codimension, Amer. J. Math. 123 (2001), 445-473. 
[25] I. N. VeKUA, “Generalized Analytic Functions”, Pergamon Press, London-Paris-Frankfurt; Addison-Wesley Publishing Co., Inc., Reading, Mass. 1962.

\author{
Université Joseph Fourier \\ 100 rue des Maths \\ 38402 Saint Martin d'Hères, France \\ herve.gaussier@ujf-grenoble.fr \\ Jae-Cheon.Joo@ujf-grenoble.fr
}

